\title{
Genome Resource of a Hypervirulent Strain C9-3 of Xanthomonas oryzae pv. oryzae Causing Bacterial Blight of Rice
}

\author{
Xiaolin Chen, ${ }^{1}$ Qili Li, ${ }^{1}$ Jinkai Wang, ${ }^{2}$ Yu Zhang, ${ }^{2}$ Lihua Tang, ${ }^{1}$ Suiping Huang, ${ }^{1}$ Jianyou Mo, ${ }^{1}$ \\ and Tangxun Guo ${ }^{1, \dagger}$ \\ ${ }^{1}$ Guangxi Key Laboratory of Biology for Crop Diseases and Insect Pests, Plant Protection Research \\ Institute, Guangxi Academy of Agricultural Sciences, Nanning 530007, Guangxi, China \\ ${ }^{2}$ State Key Laboratory for Conservation and Utilization of Subtropical Agro-bioresources, College of \\ Life Science and Technology, Guangxi University, Nanning 530004, Guangxi, China
}

\begin{abstract}
Xanthomonas oryzae pv. oryzae is the causal agent of bacterial blight, one of the most devastating diseases of rice. Here, a hypervirulent strain, C9-3, defeating Xa1, Xa10, $x a 13$, and Xa23 resistance genes, was used to extract genomic DNA for single molecule real-time (SMRT) sequencing. After assembly, the genome consists of a single-circular chromosome with the size of $4,924,298$ bp with $\mathrm{G}+\mathrm{C}$ content of $63.7 \%$ and contains 4,715 genes. Annotation and analysis of the TALE genes using a suite of applications named AnnoTALE suggested that 17 transcription activator-like effectors, including 15 typical TALEs and 2 iTALEs/truncTALEs, were encoded in the genome. The approach and genome resource will contribute to the discovery of new virulence effectors and understanding on rice- $X$. oryzae pv. oryzae interactions.
\end{abstract}

\section{Genome Announcement}

The pathogen Xanthomonas oryzae pv. oryzae is the causal agent of bacterial blight, one of the most devastating diseases of rice, which is an economically important crop (NiñoLiu et al. 2006). Typically, $X$. oryzae pv. oryzae enters through hydathodes or wounds and travels through the xylem tissue, resulting in necrotic lesions along the leaf margins following veins down the center of the leaf. Like many other gram-negative pathogenic bacteria, $X$. oryzae pv. oryzae depends on type III secretion system (T3SS) to secrete multiple effector proteins to modulate host cell functions for the benefit of the invasion process (Feng and Zhou 2012). Widespread in Xanthomonas, transcription activator-like effectors (TALEs) constitute the largest T3SS effector family and function as a host gene-specific transcriptional factor that can target both susceptibility $(S)$ and/or resistance $(R)$ genes (White et al. 2009). TALEs possess a highly repetitive DNA-binding domain of 34 amino acid repeats (Boch and Bonas 2010). Each repeat contains the variable 12th and 13th amino acids (termed 'RVD' for repeat-variable di-residue) acting as the determinants of base-recognition specificity (Scholze and Boch 2011). Each TALE also has a conserved N-terminal T3SS signal, and a C-terminus containing nuclear localization signal (NLS) and an acidic transcription activation domain (AD) (Scholze and Boch 2011). TALEs comprise 17 repeats on average, plus a final repeat truncated at 20 aa, whereas some contain $>30$ repeats (Boch and Bonas 2010). Among them, several TALEs are referred to as major TALEs of different strains of $X$. oryzae pv. oryzae that have major contributions to virulence, such as PthXo1, PthXo2, PthXo3,

${ }^{\dagger}$ Corresponding author: T. X. Guo; gtx6530@163.com

*The $e$-Xtra logo stands for "electronic extra" and indicates that supplementary tables are published online.

The author(s) declare no conflict of interest.

Accepted for publication 29 September 2021.

\section{Funding}

This work was supported by the National Natural Science Foundation of China (32160039), the Natural Science Foundation of Guangxi (2015GXNSFBA139106, 2018GXNSFAA294110); Foundation of Guangxi Key Laboratory of Biology for Crop Diseases and Insect Pests (2019-ST-01, 20-065-30-ST-06).

\section{Keywords}

Xanthomonas oryzae pv. oryzae, single molecule real-time (SMRT) sequencing, transcription activator-like effector 


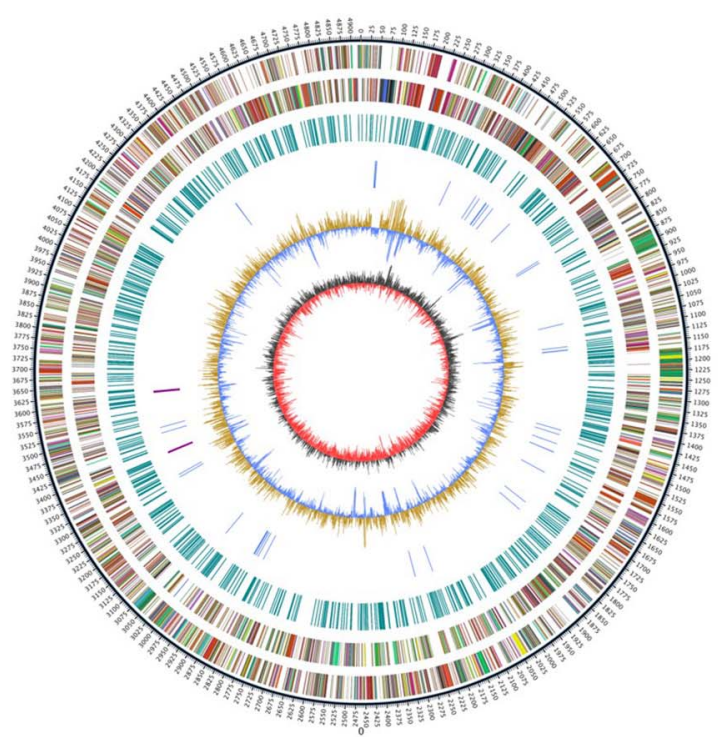

Genome size $(\mathrm{Mb})$

Scaffold number

1

$\mathrm{G}+\mathrm{C}$ content $(\%)$

63.7

Genes

4715

rRNA

6

tRNA

54

ncRNA

175

Genes assigned to COGs

3305

Genes assigned to KEGG

1989

TALE number

17

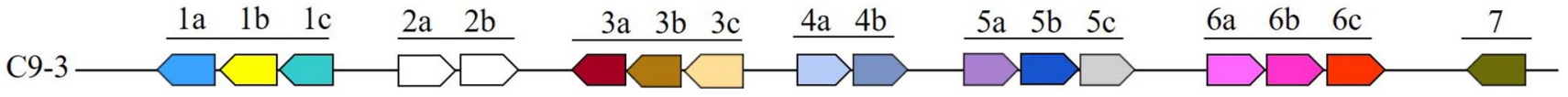

TALE gene cluster

Fig. 1. Genome features of Xanthomonas oryzae pv. oryzae C9-3. From outside to the center: the outermost circle is the chromosome with each scale of $5 \mathrm{~Kb}$; the second and third circles are forward and reverse coding DNA sequences, in which COG categories differ in color; the fourth are the repeat sequences; the fifth shows tRNA (shown in blue) and rRNA (shown in purple); the sixth and the innermost circle show GC content the GC skew. GC ratio takes the genomic mean GC ratio value as baseline, with higher-than-average values in pale yellow and lowerthan-average values in blue. $G$ content value is higher than $C$ in dark pale and lower in red. The image was created using the software Circos.

AvrXa7, TalC, and so on (Antony et al. 2010; Streubel et al. 2013; Tran et al. 2018; Yang and White 2004; Yang et al. 2006; Yu et al. 2011). Here, we report the whole genome sequence of a Chinese hypervirulent $X$. oryzae pv. oryzae strain, C9-3, which is compatible with Xa1, Xa10, xa13, and Xa23, which mediate resistance associated with the TALEs in the interaction of rice-X. oryzae pv. oryzae (Jiang et al. 2020).

X. oryzae pv. oryzae strain C9-3, one train of pathotype 2 in Chen et al. (2019), was isolated from rice in Guangxi Zhuang Autonomous Region, South China, and was hypervirulent on a series of the near-isogenic lines (NILs) of rice harboring different single resistance genes. Genomic DNA was extracted using QIAGEN Genomic-tip and was sheared using the Covaris G-Tube to generate 20-kb fragments. The sheared DNA was converted into a SMRTbell library using the SMRTbell Template Preparation kit 1.0 (Pacific Biosciences, Menlo Park, CA). After size selection using BluePippin and purification step using a $0.5 \times$ AMPure PB magnetic bead, the SMRTbell library templates were sequenced using standard SMRT sequencing on the Pacific Biosciences (PacBio) RSII system (Biomarker Technologies Corporation, Beijing, China). The de novo assembly was performed using Canu, version 1.5 (with default parameters) (Chin et al. 2013; Koren et al. 2017). Pilon version 1.21 (with default parameters) was used to polish and assess the assembly quality. Gene prediction was performed using Prodigal v.2.6 (Hyatt et al. 2010), and tRNA and rRNA were analyzed with tRNAscan-SE and Infernal 1.1 software (Lowe and Eddy 1997; Nawrocki and Eddy 2013). NCBI Prokaryotic Genome Annotation Pipeline version 4.9 was used for functional annotation of genes (Haft et al. 2018). SignalP 4.0 was used to identify genes with predicted signal peptides (Petersen et al. 2011). AnnoTALE 1.4.1 was used to detect and annotate TALE genes (Grau et al. 2016).

A total of 185,120 subreads were obtained, consisting of $2,061,927,765$ bases in total. Mean coverage for the genome was 419x. The genome was assembled into a contig $4,924,298$ bp in size, with $\mathrm{G}+\mathrm{C}$ content of $63.7 \%$. The genome contains 4,715 genes, 6 rRNAs, and 54 tRNAs. Of the 4,715 predicted genes, 3,305 and 1,989 were assigned to the COGs and KEGG databases, respectively. The number of the protein with signal peptide was 466 (Supplementary Table S1). The genome feature is shown in Figure 1. 
Table 1. Repeat variable di-residue (RVD) sequences of Xanthomonas oryzae pv. oryzae strain C9-3

\begin{tabular}{|c|c|c|c|}
\hline TALE class & TALE gene & Repeats & RVD sequence \\
\hline TalFX & tal1a & 30.5 & $\begin{array}{l}\text { NI-HG-NI-HG-NI-NI-NI-HD-NN-HD-HD-HD-NG-HD-N*-NI-HD-NN-NS-NI-NN-NN-NG- } \\
\text { NN-NN-NG-NN-HD-N*-NS-N* }\end{array}$ \\
\hline TalAS & $t a l 1 b$ & 26.5 & $\begin{array}{l}\text { NI-HG-NI-NI-HG-HD-NN-HD-HD-HD-NI-NI-NN-NI-HD-NS-HD-HG-NN-NN-HD-NS- } \\
\text { NN-HD-NG-NS-N* }\end{array}$ \\
\hline TalAG & tal1c & 19.5 & NI-NG-NN-NG-NK-NG-NI-NN-NI-NN-NI-NN-NS-NG-NS-NN-NI-N*-NS-NG \\
\hline TalAl & tal2a & 7.5 & NS-HD-NG-HD-NI-HD-NN-N* \\
\hline TalAl & $t a / 2 b$ & 17.5 & NS-HD-NG-NG-NG-NG-HD-HD-HD-HD-NN-HD-NG-HD-NI-HD-NN-N* \\
\hline TalAR & tal3a & 22.5 & NI-H*-NI-NN-NN-NN-NN-NN-HD-NI-NS-HG-HD-NI-N*-NS-NI-NI-HD-HD-N*-NS-N* \\
\hline TalAA & $t a / 3 b$ & 19.5 & NI-HG-NI-NG-HG-HD-NS-NG-HD-NN-NG-HG-NG-HD-HG-HD-HD-NI-NN-NG \\
\hline TalAH & tal3c & 19.5 & NI-N*-NI-NS-NN-NG-NN-NS-N*-NS-NN-NS-N*-HD-HG-HD-NI-HD-HD-NG \\
\hline TalAF & tal4a & 15.5 & NI-NN-NN-NI-NI-NI-HD-NS-HG-NN-NN-NN-NI-NI-NG-HD \\
\hline TalAN & $t a l 4 b$ & 20.5 & NI-N*-NI-HG-NI-NI-NS-HD-NN-HD-NS-NG-SS-HD-NI-NI-NN-NI-NN-NI-NG \\
\hline TalAD & tal5a & 23.5 & $\begin{array}{l}\text { NN-HD-NS-NG-HD-NN-N*-NI-HD-NS-HD-NN-HD-NN-HD-NN-NN-NN-NN-NN-NN- } \\
\text { NN-HD-NG }\end{array}$ \\
\hline TalAB & tal5b & 17.5 & NI-HG-NI-NI-NI-NN-HD-NS-NN-NS-NN-HD-NN-NI-HD-NN-NS-NG \\
\hline TalAL & tal5c & 17.5 & NI-NS-HD-NG-NS-NN-HD-N*-NN-NN-NI-NN-HD-HG-HD-HD-NN-NG \\
\hline TalAP & tal6a & 19.5 & HD-HD-HD-NG-N*-NN-HD-HD-N*-NI-NI-NN-HD-HI-ND-HD-NI-HD-NG-NG \\
\hline TalAO & tal6b & 15.5 & NI-NN-N*-NG-NS-NN-NN-NN-NI-NN-NI-NG-HD-HD-NI-NG \\
\hline TalAE & tal6c & 12.5 & NI-NN-NI-HG-HG-HD-NG-HD-HG-HD-HD-HD-NG \\
\hline TalAM (PthXo2) & tal7 & 21.5 & NI-HG-NI-NN-NN-NI-NN-HD-NI-HD-NS-NS-NS-HD-NN-HD-NG-HD-HD-HD-NG-NG \\
\hline
\end{tabular}

The nomenclature of Xanthomonas TALEs is nonuniform. They can be named by their genomic position (e.g., Tal1, Tal2, Tal3, etc., or Ta2a, Tal2b, etc. if part of the same genomic locus), their reaction in hosts (names starting with "Avr" for avirulence or "Pth" for pathogenicity), or their sequence similarity to previously identified TALEs (e.g., TalAA1, TalAA2, etc. for class TalAA using "AnnoTALE" annotation). Sequence analysis using AnnoTALE version 1.4.1 indicated that C9-3 genome contains 17 TALEs, consisting of 15 typical TALEs and 2 iTALEs/truncTALEs encoding genes (Table 1). The 15 typical TALEs are assigned to classes TalAF, TalAN, TalAD, TalAB, TalAL, TalAD, TalAO, TalAE, TalAM, TalAH, TalAA, TalAR, TalAG, TalAS, TalFX, and the iTALE/truncTALEs are assigned to class TalAl. Among the reference isolates PXO99A, MAFF311018, and XM9 (Chien et al. 2019), C9-3 shared the largest numbers of TALEs with isolate XM9 (Supplementary Table S2) (Chien et al. 2019). The iTALE/truncTALEs Tal2b in C9-3 is consistent with Tal3b in PXO99A overcoming Xa1 (Ji et al. 2016). Tal7 is identical to PthXo2 (TalAM) of MAFF311018, which induces the susceptibility gene OsSWEET13 to defeat xa13 recessive resistance (Yang and White 2004). AvrXa23 (TalAQ class) and AvrXa10 (TalBJ class) are absent from C9-3 (Hopkins et al. 1992; Wang et al. 2014). Interestingly, C9-3 does not have an AvrXa7 (TalAC class) or an AvrXa7 homolog (TalDV class) (Supplementary Table S3) but is incompatible with IRBB7 carrying resistance gene Xa7 (Chen et al. 2019). Tal1a of C9-3 is assigned to class TalFX and identical to Tal6c of strain XM9 which is closest to AvrXa7 (Chien et al. 2019). This observation suggested that Tal1a in C9-3 might have evolved from AvrXa7 and retain its avirulence. The genome resource is useful for the identification of effectors, pathogen-host interaction, and population genetic diversity.

The complete genome sequence of $X$. oryzae pv. oryzae C9-3 has been deposited at GenBank under the accession number CP060817. The BioProject and BioSample numbers are PRJNA659052 and SAMN15896830, respectively.

\section{Literature Cited}

Antony, G., Zhou, J., Huang, S., Li, T., Liu, B., White, F., and Yang, B. 2010. Rice xa13 recessive resistance to bacterial blight is defeated by induction of the disease susceptibility gene Os-11N3. Plant Cell 22:3864-3876.

Boch, J., and Bonas, U. 2010. Xanthomonas AvrBs3 family-type III effectors: discovery and function. Annu. Rev. Phytopathol. 48:419-436.

Chen, X. L., Wei, S. H., Yan, Q., Huang, F. K., Ma, Z. F., Li, R. F., Cen, Z. L., Yan, W. H., and Li, K. H. 2019. Virulence and DNA fingerprinting analysis of
Xanthomonas oryzae pv. oryzae identify a new pathotype in Guangxi, South China. J. Basic Microbiol. 59:1082-1091.

Chien, C. C., Chou, M. Y., Chen, C. Y., and Shih, M. C. 2019. Analysis of genetic diversity of Xanthomonas oryzae pv. oryzae populations in Taiwan. Sci. Rep. 9:316.

Chin, C. S., Alexander, D. H., Marks, P., Klammer, A. A., Drake, J., Heiner, C., Clum, A., Copeland, A., Huddleston, J., Eichler, E. E., Turner, S. W., and Korlach, J. 2013. Nonhybrid, finished microbial genome assemblies from long-read SMRT sequencing data. Nat. Methods 10:563-569. 
Feng, F., and Zhou, J. M. 2012. Plant-bacterial pathogen interactions mediated by type III effectors. Curr. Opin. Plant Biol. 15:469-476.

Grau, J., Reschke, M., Erkes, A., Streubel, J., Morgan, R. D., Wilson, G. G., Koebnik, R., and Boch, J. 2016. AnnoTALE: bioinformatics tools for identification, annotation, and nomenclature of TALEs from Xanthomonas genomic sequences. Sci. Rep. 6:21077.

Haft, D. H., DiCuccio, M., Badretdin, A., Brover, V., Chetvernin, V., O'Neill, K., et al. 2018. RefSeq: an update on prokaryotic genome annotation and curation. Nucleic Acids Res. 46:D851-D860.

Hopkins, C. M., White, F. F., Choi, S. H., Guo, A., and Leach, J. E. 1992. Identification of a family of avirulence genes from Xanthomonas oryzae pv. oryzae. Mol. Plant-Microbe Interact. 5:451-459.

Hyatt, D., Chen, G. L., Locascio, P. F., Land, M. L., Larimer, F. W., and Hauser, L. J. 2010. Prodigal: prokaryotic gene recognition and translation initiation site identification. BMC Bioinformatics 11:119.

Ji, Z., Ji, C., Liu, B., Zou, L., Chen, G., and Yang, B. 2016. Interfering TAL effectors of Xanthomonas oryzae neutralize R-gene-mediated plant disease resistance. Nat. Commun. 7:13435.

Jiang, N., Yan, J., Liang, Y., Shi, Y., He, Z., Wu, Y., Zeng, Q., Liu, X., and Peng, J. 2020. Resistance genes and their interactions with bacterial blight/leaf streak pathogens (Xanthomonas oryzae) in Rice (Oryza sativa L.)-an updated review. Rice (N. Y.) 13:3.

Koren, S., Walenz. B. P., Berlin, K., Miller, J. R., Bergman, N. H., and Phillippy, A. M. 2017. Scalable and accurate long-read assembly via adaptive $k$-mer weighting and repeat separation. Genome Res. 27:722-736.

Lowe, T. M., and Eddy, S. R. 1997. tRNAscan-SE: a program for improved detection of transfer RNA genes in genomic sequence. Nucleic Acids Res. 25:955-964.

Nawrocki, E. P., and Eddy, S. R. 2013. Infernal 1.1: 100-fold faster RNA homology searches. Bioinformatics 29:2933-2935.

Niño-Liu, D. O., Ronald, P. C., and Bogdanove, A. J. 2006. Xanthomonas oryzae pathovars: Model pathogens of a model crop. Mol. Plant Pathol. 7:303-324.
Petersen, T. N., Brunak, S., von Heijne, G., and Nielsen, H. 2011. SignalP 4.0: discriminating signal peptides from transmembrane regions. Nat. Methods 8 : 785-786.

Scholze, H., and Boch, J. 2011. TAL effectors are remote controls for gene activation. Curr. Opin. Microbiol. 14:47-53.

Streubel, J., Pesce, C., Hutin, M., Koebnik, R., Boch, J., and Szurek, B. 2013. Five phylogenetically close rice SWEET genes confer TAL effector-mediated susceptibility to Xanthomonas oryzae pv. oryzae. New Phytol. 200:808-819.

Tran, T. T., Pérez-Quintero, A. L., Wonni, I., Carpenter, S. C. D., Yu, Y., Wang, L., Leach, J. E., Verdier, V., Cunnac, S., Bogdanove, A. J., Koebnik, R., Hutin, M., and Szurek, B. 2018. Functional analysis of African Xanthomonas oryzae pv. oryzae TALomes reveals a new susceptibility gene in bacterial leaf blight of rice. PLoS Pathog. 14:e1007092.

Wang, C. L., Qin, T. F., Yu, H. M., Zhang, X. P., Che, J. Y., Gao, Y., Zheng, C. K., Yang, B., and Zhao, K. J. 2014. The broad bacterial blight resistance of rice line CBB23 is triggered by a novel transcription activator-like (TAL) effector of Xanthomonas oryzae pv. oryzae. Mol. Plant Pathol. 15: 333-341.

White, F. F., Potnis, N., Jones, J. B., and Koebnik, R. 2009. The type III effectors of Xanthomonas. Mol. Plant Pathol. 10:749-766.

Yang, B., Sugio, A., and White, F. F. 2006. Os8N3 is a host diseasesusceptibility gene for bacterial blight of rice. Proc. Natl. Acad. Sci. USA 103: 10503-10508.

Yang, B., and White, F. F. 2004. Diverse members of the AvrBs3/PthA family of type III effectors are major virulence determinants in bacterial blight disease of rice. Mol. Plant-Microbe Interact. 17:1192-1200.

Yu, Y., Streubel, J., Balzergue, S., Champion, A., Boch, J., Koebnik, R., Feng, J., Verdier, V., and Szurek, B. 2011. Colonization of rice leaf blades by an African strain of Xanthomonas oryzae pv. oryzae depends on a new TAL effector that induces the rice nodulin-3 Os11N3 gene. Mol. Plant-Microbe Interact. 24: 1102-1113. 